\title{
Arterial Decellularized Scaffolds Produced Using an Innovative Automatic System
}

\author{
Alessandro F. Pellegata ${ }^{a}$ Tommaso Dominioni ${ }^{c}$ Filippo Ballo $^{a}$ \\ Silvia Maestroni ${ }^{b}$ M. Adelaide Asnaghi ${ }^{a}$ Gianpaolo Zerbini ${ }^{b}$ Sandro Zonta ${ }^{c}$ \\ Sara Mantero ${ }^{a}$ \\ a Department of Chemistry, Materials and Chemical Engineering 'Giulio Natta', Politecnico di Milano, and \\ ${ }^{b}$ Complications of Diabetes Unit, Division of Metabolic and Cardiovascular Sciences, San Raffaele Scientific Institute, \\ Milan, and ${ }^{\mathrm{C}}$ General Surgery I, Fondazione IRCCS pol. San Matteo, Pavia, Italy
}

\section{Key Words}

Scaffold · Endothelial cell · Extracellular matrix · Artery .

Tissue engineering $\cdot$ Decellularization

\begin{abstract}
There is still an unmet clinical need for small-caliber artery substitution. Decellularized scaffolds in tissue engineering represent a promising solution. We have developed an innovative system for the automatic decellularization of blood vessels, used to process pig arteries. The system is able to automatically drive a decellularization process in a safe and reliable environment, with complex time patterns, using up to three different decellularization solutions, and providing at the same time a physical stress to improve the decellularization. The decellularization of pig arteries was evaluated by means of histology, DNA quantification and mechanical testing. Outcomes showed scaffolds with no cellular or nuclear remnants and a well-preserved tissue structure, corroborated by mechanical properties similar to native tissue. Decellularized scaffolds were seeded on the inner layer with human endothelial cells and implanted as iliac artery replacement in 4 pharmacologically immune-compromised pigs. This chimeric model was performed as a very preliminary evaluation to investigate the performances of these scaf-
\end{abstract}

folds in vivo, and to investigate the fate of seeded cells. Recipients were sacrificed on day 14 and day 70 after surgery, and vessels were found to be patent and with no evidence of thrombi formation. The inner layer was covered by endothelial cells, and the migration of cells positive for a-smoothmuscle actin was observed from the outer layer towards the tunica media. Intriguingly, the endothelial cells on explanted vessels were entirely derived from the host while the seeded cells were lost. In conclusion, this work presents a novel tool for a safe and controlled production of arterial scaffolds, with good decellularization outcomes and a good performance in a short-term, large-animal implantation.

\section{Abbreviations used in this paper}

a-SMA $\quad$-smooth-muscle actin

DAPI 4',6-diamidino-2-phenylindole

ECM extracellular matrix

hIAEC human iliac artery endothelial cells

OCT optimal cutting temperature

PBS phosphate-buffered saline

POM polyoxymethylene

vWF von Willebrand factor

\section{KARGER}

E-Mail karger@karger.com

www.karger.com/cto
(C) 2015 S. Karger AG, Basel

$1422-6405 / 15 / 2006-0363 \$ 39.50 / 0$ 


\section{Introduction}

The tissue-native extracellular matrix (ECM) represents the best possible environment for a cell because of its biochemical signaling and its structure, promoting cell adhesion, proliferation and differentiation. In recent years, the use of the ECM as a scaffold for tissue engineering has been investigated, taking into consideration the tissue-specific biological and mechanical properties of the matrix [Badylak et al., 2009]. In order to isolate the ECM from the cells, the tissue must be decellularized. This technique consists of the complete removal of all cellular and nuclear matter from the tissue, leaving the ECM as intact as possible [Crapo et al., 2011]; the best result for this process is a trade-off between aggressiveness to remove the immunogenic material and the preservation of the structure and components of the matrix. A good balance generates decellularized scaffolds that do not elicit an immune response from the host while preserving the biological and mechanical properties of the ECM [Barnes et al., 2011]. This approach has been investigated in nearly all tissues [Wolf et al., 2012; Goh et al., 2013; Keane et al., 2013; Maghsoudlou et al., 2013; Orlando et al., 2013; Pellegata et al., 2013; Weber et al., 2013; Baiguera et al., 2014; Friedrich et al., 2014] with outstanding clinical results, e.g. in the trachea [Macchiarini et al., 2008; Asnaghi et al., 2009], bladder and blood vessels (in the setting of extrahepatic portal vein substitution) [Olausson et al., 2012].

Decellularized scaffolds have also been investigated in the field of vascular tissue engineering, aiming at filling the unmet clinical gap for small-diameter $(<6 \mathrm{~mm})$ arterial substitutes [Tresoldi et al., 2015]. Synthetic grafts still perform badly with poor patency, mainly due to the cellsurface interactions and to the mechanical mismatch that leads to intimal hyperplasia of the anastomosis-adjacent vessels. In this context, decellularized blood vessels show promising results in animal models of small-diameter arterial replacements [Dahl et al., 2011; Quint et al., 2011, 2012; Meier et al., 2014; Syedain et al., 2014].

However, decellularization is still a procedure that is largely carried out manually, and it requires different steps with multiple solutions, which means it is time-consuming and holds no assurance of reliability and reproducibility. These aspects represent major drawbacks when decellularization is being aimed at settings compliant with good manufacturing practice. In order to address these issues, we have already proposed a device for the automatic decellularization of biological tissues [Pellegata et al., 2012] and other devices have been proposed by other groups [Montoya and McFetridge, 2009; Sulli- van et al., 2012; Fercana et al., 2014; Struecker et al., 2014; Price et al., 2015].

Starting from the concept of having an automated and closed system for decellularization, developed in our previous device, we focused on arterial vessels and developed a new device specifically designed for the automatic decellularization of vascular tissue, able to house vessels with different diameters and lengths, which provide both longitudinal and radial strain generated by the distal holder, since mechanical stresses enhance the decellularization outcomes [Struecker et al., 2015].

In order to validate the functionality of this new device, pig arteries of up to $6 \mathrm{~mm}$ in diameter were decellularized and characterized biologically and mechanically. Furthermore, four vessels were implanted in a small pig model, in order to preliminarily assess the in vivo outcomes of the decellularized scaffolds for short and medium-length periods. To track the fate of the seeded endothelial cells in vitro, vessels were seeded on the lumen with human endothelial cells and the implants were performed in immune-compromised pigs. Currently, the effect of endothelialization of the graft before implantation represents a major field of investigation in blood vessel tissue engineering. Conflicting evidence is reported in the literature. On the one hand, it has been shown that seeded cells are lost within a few days after the implant [Roh et al., 2010; Hibino et al., 2011], and on the other hand, better resolution has been observed, in terms of patency, intimal hyperplasia and tissue regeneration for cell-seeded implants [Quint et al., 2011; Meier et al., 2014].

\section{Materials and Methods}

\section{Tissue Harvesting}

Abdominal aortas and carotids were obtained from Large White piglets (Sus scrofa) with a body weight of 25-30 kg, under general anesthesia and on orotracheal ventilation. After tissue harvesting, the animals were sacrificed according to standard procedure. The inner diameter of the vessels ranged from 3 to $6 \mathrm{~mm}$. The study was carried out under the approval of the Ethical Committee for Animal Experimentations of the University of Pavia (permit No. 1/2012) and following Italian laws for the care of animals. Connective tissue was removed using a scalpel and the vessel was rinsed in phosphate-buffered saline (PBS) containing $1 \%$ penicillin, $1 \%$ streptomycin and $1 \%$ amphotericin B (all from Sigma-Aldrich, Milan, Italy) in order to remove blood clots. Vessels were stored at $-80^{\circ} \mathrm{C}$ until decellularization.

\section{Automatic Decellularization Device}

An innovative device for the decellularization of blood vessels was developed to conduct the process, under full automation, in a sterile, safe and reliable environment and providing control over 

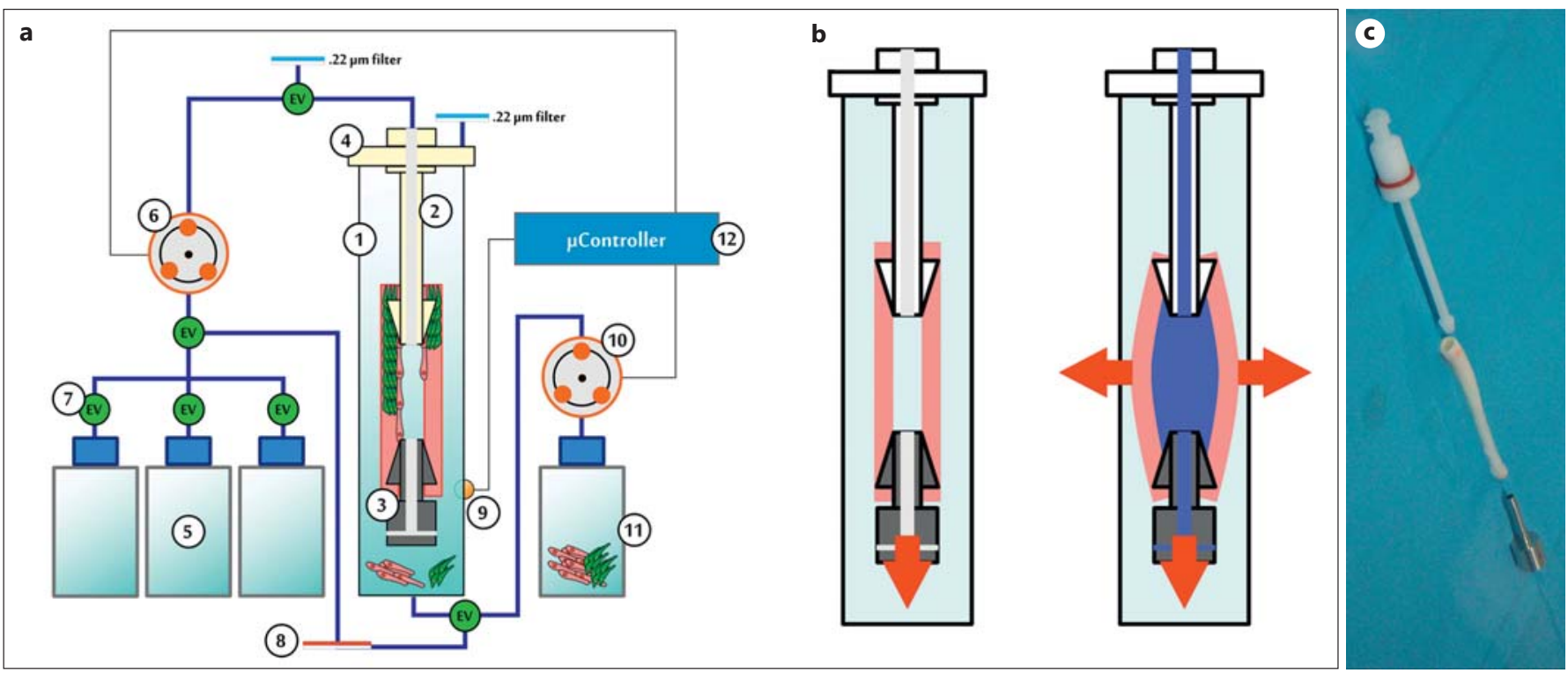

Fig. 1. a The device is composed of (1) a chamber to house the vessel by means of (2) POM proximal holding and (3) stainless-steel distal holding. The chamber is closed by (4) a POM cap provided with a venting access, (5) three reservoirs house the decellularization solution pumped inside the chamber by (6) the first pump, (7) electrovalves conduct the flows in the right direction, (8) a heat exchanger is positioned on the recirculating line in order to control fluid temperature according to (9) sensor measurements. (10) The

the most important parameters, such as timing (filling, emptying, time point of activation of the solutions and recirculation on/off), volume, flow rate and temperature. Furthermore, it was designed to provide mechanical stimulation to the tissue in order to enhance decellularization outcomes.

The device (fig. 1a) consists of a chamber in which the tissue is housed, a hydraulic system providing the filling, emptying and recirculation of the decellularization solutions and a thermal system for temperature regulation. The overall control is provided by a microcontroller-based unit.

The chamber is a hollow cylinder made of polysulfone with hydraulic access; sterile venting is provided by a $0.22-\mu \mathrm{m}$ filter mounted on a polyoxymethylene (POM) cap. Connections between the chamber, cap and holders are provided, and equipped with silicone gaskets to avoid leakage. Inside the chamber, the vessel is housed by means of two holders (fig. 1c); the proximal one is made of POM and the distal one is made of medical-grade stainless steel. The device can house vessels of different lengths (up to 100 $\mathrm{mm})$ and diameters $(3-8 \mathrm{~mm})$. Three sizes of holder are available: small for vessel diameters of 3-4 mm, medium for 5-6 $\mathrm{mm}$ and large for 7-8 $\mathrm{mm}$.

The mechanical stimulation is provided by the distal holder (fig. 1b) based on the mechanical properties of pig blood vessels evaluated previously [Pellegata et al., 2013]. The weight of the holder was designed to provide estimated longitudinal elongations inside physiological ranges [Das et al., 2015] (table 1, elongation ranges). Furthermore, the internal geometry of the holder generates a pressure drop due to shrinkage of the flow area and a T- emptying pump pulls out decellularization solutions from the chamber to (11) the waste container, and the whole system is controlled by (12) a microcontroller. b Stress exerted by the holders. The weight of the stainless-steel distal holder exerts a longitudinal strain on the vessel (left), and its internal geometry generates a drop in pressure that promotes a cyclic radial strain on the vessel (right). c The proximal holder is made of POM (top) and the distal holder is made of AISI 316L stainless steel (bottom). junction. This drop, coupled with vessel compliance, exerts a cyclic radial deformation, estimated to be near to physiological ranges [Piola et al., 2012] (table 1, values estimated using COMSOL Multiphysics 4.4 software).

The hydraulic system is driven by two WPM2 pumps (WELCO, Tokyo, Japan), one providing the filling of the chamber and one providing the emptying and recirculation of the solutions. The tubes are made of Pharmed ${ }^{\circledR}$ BPT biocompatible tubing (Saint Gobain, Germany) and the reservoirs are made of PYREX ${ }^{\circledR}$ (VWR, Milan, Italy). Connections are provided by polypropylene luer lock connectors (Qosina, Italy) and pinch electrovalves (Sirai, Italy) control flow direction.

The thermal system consists of a Peltier cell (RS, Italy) linked to a radiator placed on the recirculation tube; a digital sensor provides the monitoring of the temperature inside the chamber, and the working range for the temperature is $18-37^{\circ} \mathrm{C}$.

The control system is based on an open-source microcontroller platform, Arduino Mega (Arduino, Italy), that drives all the operations of the device and allows the end-user to set the working parameters as the number of solutions, timings (filling, emptying, action of every solution), recirculation patterns, volumes and temperatures.

During operation, the working scheme (fig. 2) that is repeated according to the user settings is: filling of the chamber with the desired quantity of the solution and the specified flow rate, recirculation of the solution with a specified pattern of timing (recirculation on/off), flow rate and temperature and emptying of the chamber. 

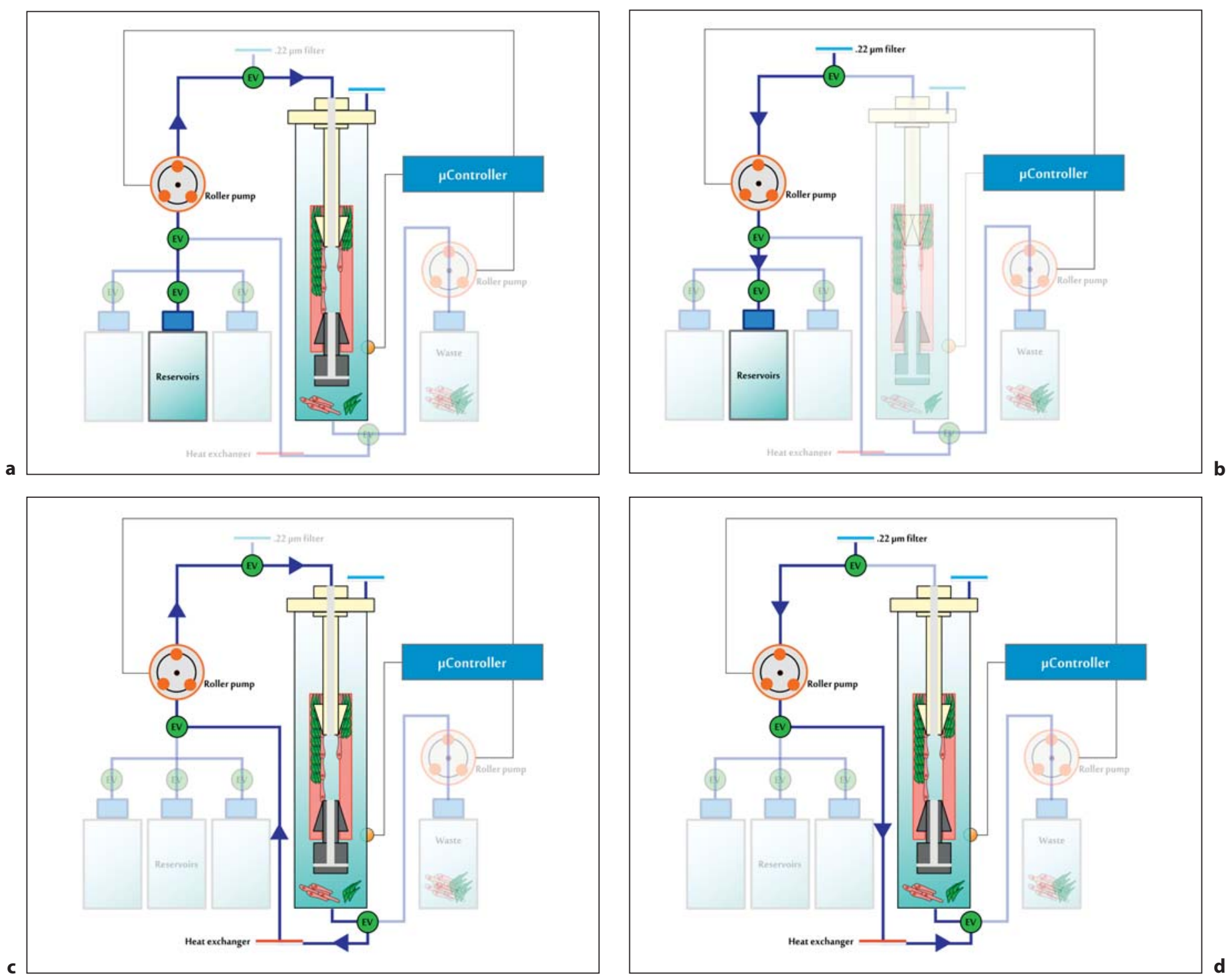

Fig. 2. Device working phases. a Filling phase in which the chamber is filled with a specific flow rate and a specific volume defined by the user. b Emptying of the filling line to avoid mixing of different decellularization solutions. c Recirculation phase in which the mechanical stimulation is applied to the vessel; the recirculation flow rate and on/off pattern can be defined by the user. $\mathbf{d}$ Emptying of the recirculation line inside the chamber. e Chamberemptying phase.

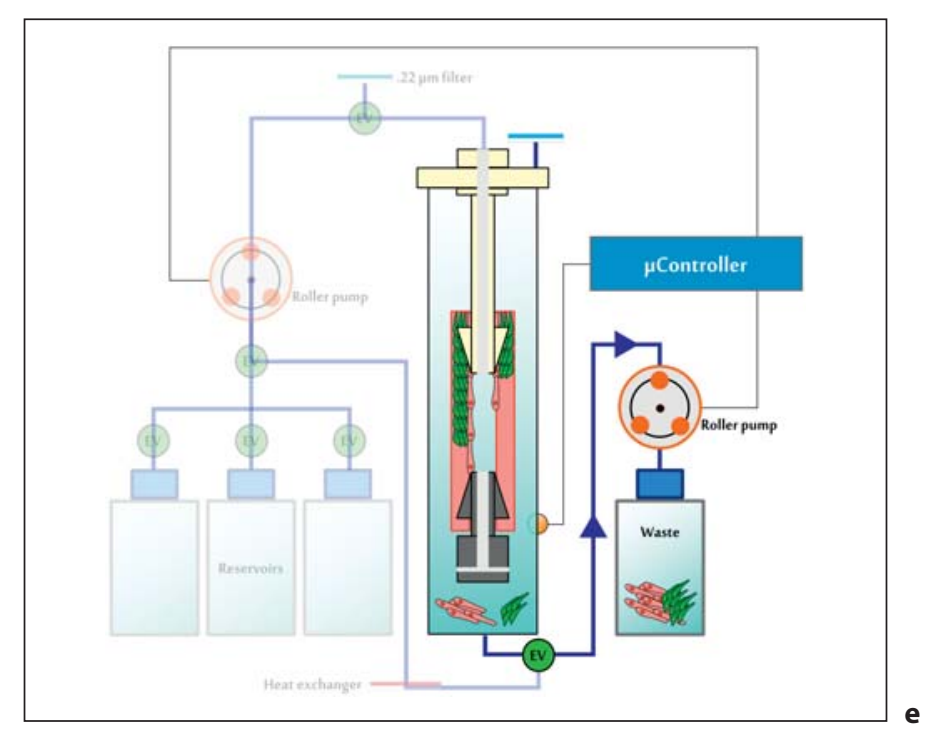




\section{Decellularization Protocol}

Six arteries with a diameter of $3 \mathrm{~mm}$ and six arteries with a diameter of $5 \mathrm{~mm}$ were processed to evaluate the decellularization outcomes and the functionality of the device as well as investigate the stimulation effect. Five arteries with a diameter of 3-6 mm were then processed under stimulation, in order to investigate the mechanical properties and four arteries with a diameter of $6 \mathrm{~mm}$ were processed and used in the animal model.

The arteries were mounted on the holders using a 4-0 silk thread tied around the vessels. Decellularization protocol was $24 \mathrm{~h}$ with a complete exchange every $2 \mathrm{~h}$ of deionized water with $1 \%$ penicillin, $1 \%$ streptomycin and $1 \%$ amphotericin B, $24 \mathrm{~h}$ with a complete exchange every $12 \mathrm{~h}$ of $4 \%$ sodium deoxycholate and $48 \mathrm{~h}$ with a complete exchange every $6 \mathrm{~h}$ of $10 \%$ fetal bovine serum in $0.9 \%$ sodium chloride. The perfusion flow rate was $40 \mathrm{ml} / \mathrm{min}$. Trials were performed by either applying the distal holder and consequently the estimated mechanical stimulations (table 1), and without stimulation. Finally, an extensive washing overnight with PBS at $4^{\circ} \mathrm{C}$, followed by 3 washings at room temperature, were performed. All reagents were from Sigma-Aldrich.

\section{Animal Model Implantation}

Four scaffolds decellularized with the device (diameter $6 \mathrm{~mm}$ and length $50 \mathrm{~mm}$ ) were seeded with $1.7 \times 10^{5} \mathrm{cells} / \mathrm{cm}^{2}$ human iliac artery endothelial cells (hIAEC; Lonza, USA) on the lumen and cultured for 3 days in complete endothelial growth medium (EGM-2, Lonza) with a $300^{\circ}$ rotation every $6 \mathrm{~h}$ to reach an even distribution of the hIAEC on the surface. The recipient animals were four $25-\mathrm{kg}$ Large White piglets. For the surgical operation, general anesthesia was administered and orotracheal ventilation was provided, a midline laparotomy was made and the left iliac artery was exposed, and then a 50-mm segment was excised and replaced with the hIAEC-seeded scaffold. Postoperatively, $0.6 \mathrm{ml} /$ day of cyclosporine was given to the animals until sacrifice on postoperative (PO) day $14(\mathrm{n}=2)$ or day $70(\mathrm{n}=2)$. No antiplatelet therapy was administered during the study.

\section{Histological Analysis on Decellularized Scaffolds}

Native and decellularized vessels were fixed for $24 \mathrm{~h}$ in $4 \%$ paraformaldehyde and dehydrated in PBS -/- glucose $40 \%$, embedded in optimal cutting temperature (OCT) compound (Sakura, USA). OCT compound blocks were cut into 8 - $\mu \mathrm{m}$ sections and stained with hematoxylin and eosin or 4',6-diamidino-2-phenylindole (DAPI) according to the manufacturer's instructions. Images were captured using an AxioImager A1 microscope (Zeiss, Germany).

\section{Immunofluorescence on Seeded Scaffolds and Explanted}

Vessels

Decellularized scaffolds and explanted vessels were fixed for $24 \mathrm{~h}$ in $4 \%$ paraformaldehyde and dehydrated in PBS -/- glucose $40 \%$, embedded in OCT compound, snap-frozen in a mixture of isopentane and liquid nitrogen and stored at $-80^{\circ} \mathrm{C}$. Subsequently, $5-\mu \mathrm{m}$ vessel sections were placed on slides and stored at $-20^{\circ} \mathrm{C}$. Frozen sections, fixed in cold acetone, were stained according to standard techniques. Images were captured using an AxioImager A1 microscope. As a negative control, the primary antibody was either substituted with control immunoglobulins or omitted. The following primary antibodies were used: mouse monoclonal anti-human (h)CD31 (Dako, Denmark), mouse monoclonal anti-
Table 1. Geometry and physical effects of the distal holder

\begin{tabular}{llllll}
\hline $\begin{array}{l}\text { Inlet } \mathrm{D}, \\
\mathrm{mm}\end{array}$ & $\begin{array}{l}\text { Outlet } \mathrm{D}, \\
\mathrm{mm}\end{array}$ & $\begin{array}{l}\text { Weight, Estimated } \\
\varepsilon_{\text {long }} \%\end{array}$ & $\begin{array}{l}\text { Maximum estimated } \\
\text { pressure drop at } \\
40 \mathrm{ml} / \mathrm{min}, \mathrm{Pa}\end{array}$ & $\begin{array}{l}\text { Maximum } \\
\text { estimated } \\
\varepsilon_{\text {circ }} \%\end{array}$ \\
\hline 2 & 0.5 & 2.15 & 8 & 6,511 & 10.00 \\
4 & 0.5 & 4.10 & 9.2 & 5,468 & 10.40 \\
6 & 0.54 & 6.70 & 9.7 & 5,333 & 11.40 \\
\hline
\end{tabular}

Inlet, outlet, weight, pressure drop and estimated elongations related to the distal holder and its effect. Pressure drop was calculated with a computational fluid dynamic model and the related estimated circumferential deformation was calculated with a computational structure mechanic model, both processed in Comsol Multiphysics 4.4. D = Diameter; $\varepsilon_{\text {circ }}=$ estimated circumferential deformation; $\varepsilon_{\text {long }}=$ estimated longitudinal elongation.

pig CD31 (AbD Serotec, UK), rabbit polyclonal anti-von Willebrand factor (vWF; Dako), mouse monoclonal anti a-smooth muscle actin (Sigma-Aldrich). Isotope-matched secondary antibodies, either TRITC- or FITC-conjugated (all from Jackson, USA), were used along with DAPI counterstaining (Sigma-Aldrich).

\section{DNA Quantification}

Samples of vessels were excised for DNA quantification, dried, weighted and lysed using a lysing buffer $(10 \mathrm{mM} \mathrm{NaCl}, 10 \mathrm{~mm}$ Tris, $10 \mathrm{mM}$ EDTA, $0.5 \%$ SDS and $0.1 \mathrm{mg} / \mathrm{ml}$ proteinase $\mathrm{K}$ ) overnight at $55^{\circ} \mathrm{C}$. For protein precipitation, $3 \mathrm{M}$ sodium acetate was used, and finally DNA was precipitated using 98 and $70 \%$ ethanol and resuspended in Tris-EDTA buffer. DNA was marked using SYBRGreen according to the manufacturer's protocol. Fluorescence was measured using a spectrophotometer (Genios, Tecan, Switzerland) at an emission wavelength of $535 \mathrm{~nm}$ (excitation $485 \mathrm{~nm}$ ). All reagents were from Sigma-Aldrich.

\section{Mechanical Characterization}

Native and decellularized vessels (processed with stimulation) representative of the diameter range of 3-6 $\mathrm{mm}$ were cut into 5 - $\mathrm{mm}$-wide, ring-shaped specimens (native $\mathrm{n}=9$ and decellularized $n=15$ ) for evaluation of compliance and burst pressure. Samples were mounted on a Synergie $200 \mathrm{H}$ uniaxial testing machine (MTS, USA) and parameters were acquired using the TestWorks software (MTS, USA). A preloading of $0.015 \mathrm{~N}$ was imposed and the sample length was reported. To evaluate the compliance, 16 loading-unloading cycles were performed at an actuator speed of $1 \mathrm{~mm} / \mathrm{s}$ and a load end point of $0.8 \mathrm{~N}$. Then, a loading ramp was imposed at a speed of $1 \mathrm{~mm} / \mathrm{s}$ until failure of the vessel, in order to calculate the burst pressure. According to Laplace's law, an equivalence criterion between the force acting on the specimen and the pressure was set. The compliance was calculated as:

$$
C\left[\frac{1}{\mathrm{~mm} \mathrm{Hg}}\right]=\frac{\frac{D_{120}-D_{80}[\mathrm{~mm}]}{D_{0}[\mathrm{~mm}]}}{120-80[\mathrm{~mm} \mathrm{Hg}]} .
$$


Table 2. Statistical values of the mechanical properties

\begin{tabular}{llll}
\hline Mechanical properties & 25th percentile & Median & 75th percentile \\
\hline $\begin{array}{llll}\text { Compliance, } 1 / \mathrm{mm} \mathrm{Hg} \\
\quad\end{array}$ & 0.001770 & 0.002190 & 0.002545 \\
$\quad$ Native vessels & 0.001972 & 0.002131 & 0.002647 \\
$\quad$ Decellularized vessels & & & \\
Burst pressure, mm Hg & 1,886 & 2,331 & 2,657 \\
$\quad$ Native vessels & 1,526 & 2,508 & 3,782 \\
$\quad$ Decellularized vessels & & & \\
\hline
\end{tabular}

Where $D_{P}$ is the diameter corresponding to pressure $P$ and calculated by an equivalence between the diameter itself and the length of the specimen:

$$
D=\frac{2}{\pi} l \text {. }
$$

In order to calculate the burst pressure, from the failure test, the pressure was derived, setting equivalence between the force acting on the specimen and the pressure itself as follows:

$$
P[\mathrm{~mm} \mathrm{Hg}]=\frac{F[\mathrm{~N}]}{w D_{\text {failure }}\left[\mathrm{mm}^{2}\right]} .
$$

Where $F$ is the force measured by the loading cell of the testing machine, $w$ is the width of the ring specimen $(5 \mathrm{~mm})$ and $D_{\text {failure }}$ is the diameter calculated with the equation (2).

\section{Ultrasound Doppler Analysis}

On PO day 14, an ultrasound scan was performed using a convex probe (Ultrasound platform, MyLab 25 Gold, CA541 convex array probe, $2-8 \mathrm{MHz}$, Esaote, Italy). Real-time B-mode scanning, together with a Doppler analysis, was completed along the native abdominal aorta and iliac arteries. The ultrasound scanning was of both iliac arteries, so the native vessel represented the interior control for the treated vessel. This scanning analyzed the following features: presence of pathological dilatation of the bioengineered vascular segment (aneurysmatic degeneration), thickening of the artery wall and the presence of endoluminal thrombosis. The Doppler analysis pointed out the presence of arterial blood flow and evaluated the pulse wave profile.

\section{Statistics}

Statistical analysis was performed using the Mann-Whitney nonparametric test. A p value of 0.05 was considered the significance limit. Analyses were made using GraphPad Prism (GraphPad Software Inc., USA). Results are expressed as median, quartiles, minimum and maximum values.

\section{Results}

Pig blood vessels were decellularized using the innovative device for automated decellularization. To assess the decellularization outcomes, these vessels were analyzed on histology and by DNA quantification and mechanical characterization. No residual cells nor nuclear material were visible by hematoxylin and eosin staining, while the concentric structure of elastic laminae that characterizes the arterial tissue was intact, with no damage or evident signs of degradation (fig. 3a). The absence of nuclear residues was also confirmed by the DAPI staining (fig. 3c, d). Moreover, the DNA content in the decellularized tissue, representing the key marker of good decellularization outcomes, showed levels $<50 \mathrm{ng} / \mathrm{mg}$ of dry weight threshold for vessels of both 3 and $5 \mathrm{~mm}$ in diameter when the mechanical stimulation was applied; without mechanical stimulation, the residual DNA quantity for the 3 - $\mathrm{mm}$ vessels was over the threshold (fig. 3i). The DNA residual quantity between different vessels of the same group ( $3 \mathrm{~mm}$ with stimulation, $3 \mathrm{~mm}$ without stimulation, $5 \mathrm{~mm}$ with stimulation and $5 \mathrm{~mm}$ without stimulation) showed no statistically significant difference (fig. 3j).

Concerning the mechanical properties, ring-shaped specimens were characterized using an uniaxial testing machine and values were derived using a mathematical model. The decellularized vessels showed no statistically significant differences with respect to native tissue for both compliance and burst pressure (fig. 3g, h; table 2).

In order to investigate the in vivo behavior of the decellularized scaffolds over a short and medium term, four scaffolds were seeded with hIAEC and implanted as iliac artery substitutions in pig recipients, two for investigating implantation at 2 weeks and two at 10 weeks ( 1 pig of the 10-week group died from causes not related to the vascular implant). The hIAEC were characterized in vitro on tissue culture polystyrene as positive for $\mathrm{hCD} 31$ (fig. 4a). After seeding the hIAEC on the scaffolds and 3 days of static culture, endothelial cells positive for vWF and hCD31 covered the lumen (fig. 4b). Concerning the group sacrificed on PO day 14, the two grafts were found to be patent without any sign of thrombi formation; the vessels showed a near-confluent endothelium as revealed by cells positive for vWF on the lumen (fig. 4c). Moreover, from the outer side of the vessels, there was a migra- 

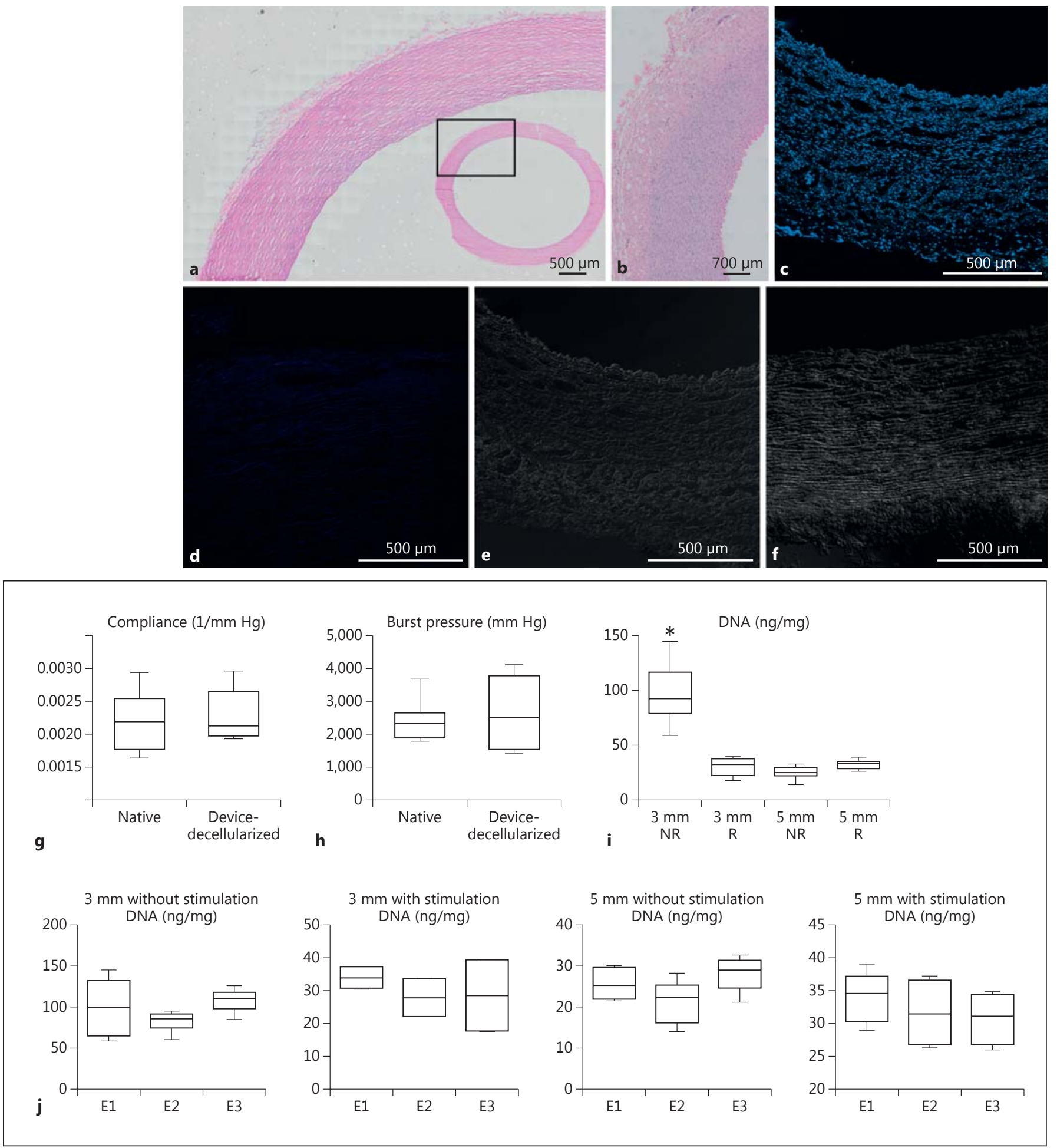

Fig. 3. Analysis of decellularized vessels. Decellularized (a) and native (b) vessels stained with HE. DAPI (c) and brightfield (e) staining of native vessels. DAPI (d) and brightfield (f) staining of decellularized arterial tissue. Compliance $(\mathbf{g})$ and burst pressure $(\mathbf{h})$ of native and decellularized vessels $3-6 \mathrm{~mm}$ in diameter. i Overall residual DNA content in the decellularized vessels with (R) or without mechanical stimulation (NR); DNA native quantity median was 2,564 ng/mg (1st quartile 2,144 and 3rd quartile 2,987). j Details of the single-vessel residual DNA quantity for the assessment of repeatability of the device. ${ }^{*} \mathrm{p}<0.05$. 

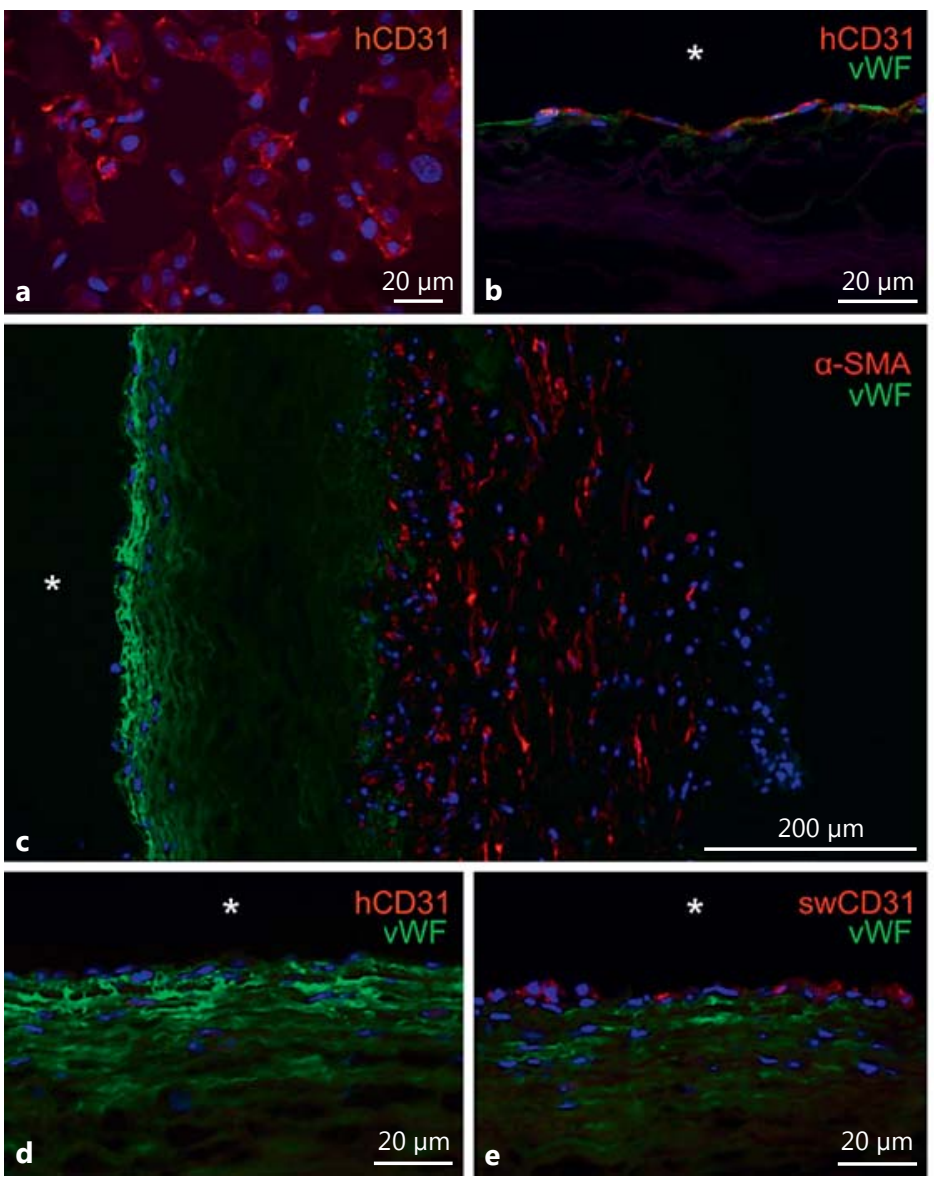

VWF

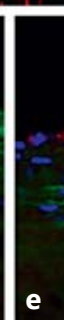

*

swCD31 VWF

Fig. 4. Decellularized vessels implanted in pigs. a hIAEC stained positive for hCD31 (red) on tissue culture polystyrene. b After 3 days of culture, hIAEC seeded on the lumen of the pig decellularized arterial scaffold were positive for vWF (green) and hCD31 (red). c Decellularized scaffold seeded with hIAEC implanted in vivo in a pig model for 14 days. The vessel was patent with no signs of thrombi formation and the lumen was populated by vWF-positive cells (green), while from the outer tunica there was a migration of a-SMA-positive cells (red). d Cells on the lumen were negative for hCD31 (red). e Cells on the lumen were positive for pig
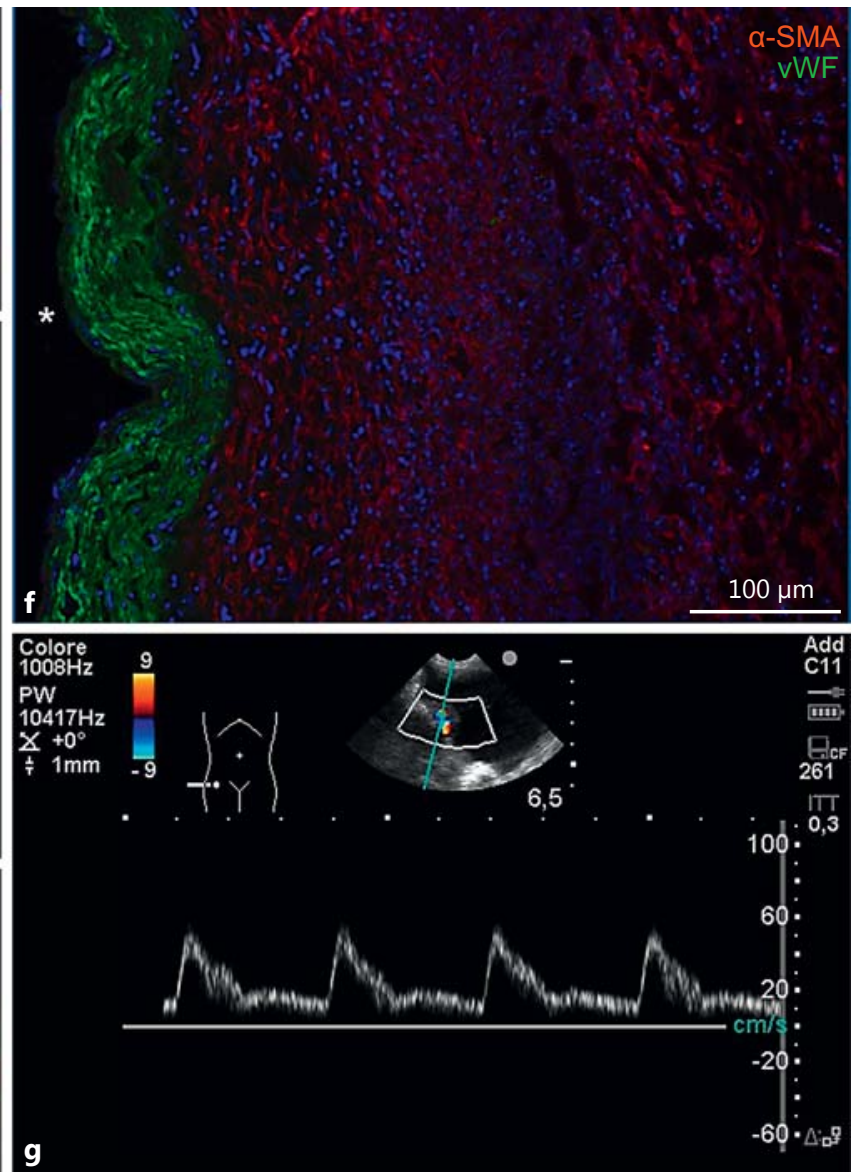

CD31 (red). f Decellularized scaffold seeded with hIAEC implanted in vivo in a pig model for 10 weeks. The vessel was patent and the lumen was populated by a near-confluent layer of vWF-positive cells (green) and the tunica media and adventitia were extensively populated by a-SMA-positive cells (red). g Doppler analysis of the implanted vessels. The profile of the pulsatile wave is perfectly conserved with a mean speed of propagation of $52 \mathrm{~cm} / \mathrm{s}$. In all images, nuclei are stained with DAPI (blue) and the asterisk indicates the lumen. tion of $\alpha$-smooth-muscle actin ( $\alpha$-SMA)-positive cells, suggesting the recruitment by the vessel of smooth-muscle cells and fibroblasts. Interestingly, the endothelial cells that were found on the lumen were negative for hCD31 but positive for pig CD31 (fig. $4 \mathrm{~d}$, e), suggesting that the hIAEC seeded in vitro onto the vessels were removed in vivo and substituted by host pig endothelial cells. Finally, B-mode real-time ultrasound scanning highlighted the absence of aneurysmatic dilatation or the presence of endoluminal thrombosis in the bioengineered vascular segment implanted. No echographic alterations were detect- able on the vascular wall. The Doppler scanning confirmed a preserved blood flow with a normal pulse wave profile. The comparison between the native iliac vessel with the bioengineered vascular tract showed a comparable amplitude of the pulse wave in the implanted bioengineered vessel with respect to the native contralateral iliac artery (fig. 4g). The graft explanted from the animals sacrificed after 10 weeks was also patent and showed a deeper repopulation of the tunica media and adventitia by $\alpha$-SMA-positive cells. The intima was covered by a confluent layer of vWF-positive endothelial cells (fig. 4f). 


\section{Discussion}

In recent years, decellularized tissues have been used for many relevant studies in the field of tissue engineering, in simple tissues and whole organs, and attaining outstanding clinical applications in some cases. These scaffolds have the proper biochemical and structural environment for promoting cell growth and differentiation, and their mechanical properties can support grafting and provide the right stimuli for the cells in order to engineer the tissue.

In the field of vascular tissue engineering, in particular, promising results have been obtained using decellularized scaffolds in both small [Quint et al., 2012] and large [Dahl et al., 2011; Quint et al., 2011] animal models and, more recently, in a clinical implantation [Olausson et al., 2012]. This all highlights the potential and the perspectives of these ECM scaffolds.

In the field of tissue engineering, standards require the safety, reliability and reproducibility of the process. The use of a device that autonomously drives the procedure drastically lowers the risk related to hand-operation and the variability related to different operators. Considering that the source for decellularized tissues is mainly represented by cadaveric donors, an intrinsic variability cannot be avoided, so it is crucial to reduce the variability in the process. To this end, the device presented in this paper automatically operates a decellularization process that can involve different solutions with complex time patterns.

Other study groups have addressed decellularization with various devices, but their focus has been on achieving a mean of decellularization, like perfusion, rather than automating the whole process to provide a safe and reliable environment.

The holders feature an innovation that simultaneously provides a longitudinal and a circumferential stress that act on the vessel, thanks to perfusion and the geometry of the holder. A mechanical stimulation on the longitudinal and circumferential axes of close to $10 \%$ enhances the outcome of decellularization. Indeed, our results showed how inducing a cyclic radial strain ameliorated the residual DNA quantity for vessels with a very small diameter. The safety of the device was demonstrated by intensive sterility tests. In order to assess the repeatability of the device by means of a quantitative method, we compared the residual DNA quantity among the different vessels of a same experimental condition, and demonstrated that there are no differences, therefore allowing us to conclude that the device delivers repeatable results.

Automatic Decellularization Device
The decellularizations performed by the device showed good results in terms of cell removal, residual DNA quantity and the preservation of tissue morphology. Indeed, these aspects play a key role in the implantation of the graft [Keane et al., 2012], since incomplete removal of nuclear material could elicit an excessive inflammatory process which would compromise the tissue remodeling, and a modification in the structure could lead to a loss of mechanical properties and, consequently, intimal hyperplasia or failure. The compliance and burst pressure of the decellularized scaffolds showed no statistically significant differences with respect to the native tissue, corroborating the positive outcome of decellularization. It is crucial that the protocol does not alter the structure of the ECM, because mechanical properties are strictly correlated with the morphology of the tissue. For blood vessels, in particular, the compliance should be similar to the native tissue in order to avoid a mechanical mismatch at the anastomosis site that promotes negative remodeling in the native vessels, leading to intimal hyperplasia and, finally, occlusion [Seifu et al., 2013].

The vessels decellularized using the device were also investigated in a large-animal model to preliminarily assess their in vivo functionality; pharmacologically immune-compromised pigs received iliac artery substitution transplants with decellularized scaffolds seeded with hIAEC. The vessels were found to be patent and with no signs of thrombi formation after 2 and 10 weeks. Histological immunofluorescence of the vessels revealed diffused vWF-positive cells on the lumen and the migration of a-SMA-positive cells toward the tunica media from the outer layer of the vessel at 2 weeks. In addition, an ultrasound Doppler analysis performed before sacrifice showed functional grafted blood vessels. After a mediumterm, the results confirmed the patency and showed that the cellular repopulation of the graft was significantly increased. These findings gave a preliminary assessment that decellularized vessels processed using the device can withstand the surgery and avoid short-term-related complications.

The particular approach used in our study allowed an easy identification of the seeded cells with respect to the host cells. Interestingly, we found that the endothelial cells seeded previously to the implant were not present at the time of sacrifice; this evidence matches the results reported by Roh et al. [2010]. In their study, bone marrow mononuclear cells seeded onto polymeric scaffolds and grafted in SCID/bg immune-deficient mice disappeared within a few days after the implant, and the regeneration of the graft was probably due to cells recruited within the 
adjacent vessels. The loss of hIAEC in the chimeric study presented here may have been caused by a xenorejection triggered by the innate arm of the immune system that recognizes some species-specific proteins which overcome the impairment of $\mathrm{T}$ cells provoked by the cyclosporine. However, Hibino et al. [2011] demonstrated the same process in an immune-competent mouse model where seeded cells were lost and the graft was populated by host-derived cells. Quint et al. [2011] investigated the outcome of seeded cells by using GFP-labeled endothelial cells in a pig model, and found that at PO day 30, nearly half of the seeded GFP-positive cells had been lost.

Many relevant studies [Meier et al., 2014; Tara et al., 2015] compare the outcomes of endothelial cell-seeded grafts with unseeded ones. Indeed, there is a contradiction, on the one hand, the seeded endothelial cells are lost within a few days, and on the other, it seems that cell-free implants have a worse resolution in the long term than seeded ones. Very recently, Meier et al. [2014] investigated the in vivo implantation in ovine femoral arteries of fibrin-gel decellularized engineered vessels with no cells or with blood-outgrowth endothelial cell seeding. Their findings were that the cell-seeded grafts had a better resolution than the cell-free ones, even if they concluded that the administration of antiplatelet drugs could have altered the outcomes. In comparison, our findings involve implants without antiplatelet therapy, strengthening the results of the study by Meier et al. [2014]. Our study does have limitations, however, concerning the number of animals involved and the peculiarity of the chimeric model in which the immune-suppressive drug could have influenced the outcome.
Overall, it can be speculated that seeded endothelial cells play a role in the early phases that influence the longterm outcome. This role could be paracrine signaling that promotes the recruitment of host endothelial cells or influences the immune cell response, as has been demonstrated in vitro on the polarization of macrophages towards an M2 proremodeling phenotype [He et al., 2012].

\section{Conclusion}

Decellularized scaffolds represent a promising and powerful tool for vascular tissue engineering, as demonstrated by the animal models and the first clinical outcomes. In this context, the automatic device presented in this work represents a valuable tool for the production of acellular scaffolds, the system proved to be functional and reliable and the decellularization process was effective and preserved the mechanical properties of the tissue. The decellularized scaffolds processed with the device were seeded with hIAEC implanted in immune-compromised pigs and were found to have patency on PO days 14 and 70. In conclusion, the device represents a step toward the compliance of guidelines in decellularization processes.

\section{Disclosure Statement}

The authors declare there are no conflicts of interest.

\section{References}

Asnaghi, M.A., P. Jungebluth, M. Raimondi, S. Dickinson, L.E.N. Rees, T. Go, T. Cogan, A. Dodson, P. Parnigotto, A. Hollander, M. Birchall, M. Conconi, P. Macchiarini, S. Mantero (2009) A double-chamber rotating bioreactor for the development of tissue-engineered hollow organs: from concept to clinical trial. Biomaterials 30: 5260-5269.

Badylak, S., D. Freytes, T. Gilbert (2009) Extracellular matrix as a biological scaffold material: structure and function. Acta Biomaterial 5: $1-13$.

Baiguera, S., C. Del Gaudio, E. Lucatelli, E. Kuevda, M. Boieri, B. Mazzanti, A. Bianco, P. Macchiarini (2014) Electrospun gelatin scaffolds incorporating rat decellularized brain extracellular matrix for neural tissue engineering. Biomaterials 35: 1205-1214.
Barnes, C., J. Brison, R. Michel, B. Brown, D. Castner, S. Badylak, B. Ratner (2011) The surface molecular functionality of decellularized extracellular matrices. Biomaterials 32: $137-$ 143.

Crapo, P.M., T.W. Gilbert, S.F. Badylak (2011) An overview of tissue and whole organ decellularization processes. Biomaterials 32: 32333243.

Dahl, S.L.M., A. Kypson, J. Lawson, J. Blum, J. Strader, Y. Li, R. Manson, W. Tente, L. DiBernardo, M.T. Hensley, R. Carter, T. Williams, H. Prichard, M. Dey, K. Begelman, L. Niklason (2011) Readily available tissue-engineered vascular grafts. Sci Transl Med 3: $68 \mathrm{ra9}$

Das, A., A. Paul, M.D. Taylor, R.K. Banerjee (2015) Pulsatile arterial wall-blood flow inter- action with wall pre-stress computed using an inverse algorithm. Biomed Eng Online 14(suppl 1): S18.

Fercana, G., D. Bowser, M.M. Portilla, E.M. Langan, C.G. Carsten, D.L. Cull, L.N. Sierad, D.T. Simionescu (2014) Platform technologies for decellularization, tunic-specific cell seeding, and in vitro conditioning of extended length, small diameter vascular grafts. Tissue Eng Part C Methods 20: 1016-1027.

Friedrich, L.H., P. Jungebluth, S. Sjoqvist, V. Lundin, J.C. Haag, G. Lemon, Y. Gustafsson, F Ajalloueian, A. Sotnichenko, H. Kielstein, M.A. Burguillos, B. Joseph, A.I. Teixeira, M.L. Lim, P. Macchiarini (2014) Preservation of aortic root architecture and properties using a detergent-enzymatic perfusion protocol. Biomaterials 35: 1907-1913. 
Goh, S.K., S. Bertera, P. Olsen, J.E. Candiello, W. Halfter, G. Uechi, M. Balasubramani, S.A. Johnson, B.M. Sicari, E. Kollar, S.F. Badylak, I. Banerjee (2013) Perfusion-decellularized pancreas as a natural 3D scaffold for pancreatic tissue and whole organ engineering. Biomaterials 34: 6760-6772.

He, H., J. Xu, C.M. Warren, D. Duan, X. Li, L. Wu, M.L. Iruela-Arispe (2012) Endothelial cells provide an instructive niche for the differentiation and functional polarization of M2-like macrophages. Blood 120: 3152-3162.

Hibino, N., G. Villalona, N. Pietris, D.R. Duncan, A. Schoffner, J.D. Roh, T. Yi, L.W. Dobrucki, D. Mejias, R. Sawh-Martinez, J.K. Harrington, A. Sinusas, D.S. Krause, T. Kyriakides, W.M. Saltzman, J.S. Pober, T. Shin'oka, C.K. Breuer (2011) Tissue-engineered vascular grafts form neovessels that arise from regeneration of the adjacent blood vessel. FASEB J 25: 2731-2739.

Keane, T.J., R. Londono, R.M. Carey, C.A. Carruthers, J.E. Reing, C.L. Dearth, A. D’Amore, C.J. Medberry, S.F. Badylak (2013) Preparation and characterization of a biologic scaffold from esophageal mucosa. Biomaterials 34: 6729-6737.

Keane, T.J., R. Londono, N.J. Turner, S.F. Badylak (2012) Consequences of ineffective decellularization of biologic scaffolds on the host response. Biomaterials 33: 1771-1781.

Macchiarini, P., P. Jungebluth, T. Go, M.A. Asnaghi, L. Rees, T. Cogan, A. Dodson, J. Martorell, S. Bellini, P. Parnigotto, S. Dickinson, A. Hollander, S. Mantero, M. Conconi, M. Birchall (2008) Clinical transplantation of a tissue-engineered airway. Lancet 372: $2023-$ 2030.

Maghsoudlou, P., F. Georgiades, A. Tyraskis, G. Totonelli, S.P. Loukogeorgakis, G. Orlando, P. Shangaris, P. Lange, J.M. Delalande, A.J. Burns, A. Cenedese, N.J. Sebire, M. Turmaine, B.N. Guest, J.F. Alcorn, A. Atala, M.A. Birchall, M.J. Elliott, S. Eaton, A. Pierro, T.W. Gilbert, P. De Coppi (2013) Preservation of micro-architecture and angiogenic potential in a pulmonary acellular matrix obtained using intermittent intra-tracheal flow of detergent enzymatic treatment. Biomaterials 34 : 6638-6648.

Meier, L.A., Z.H. Syedain, M.T. Lahti, S.S. Johnson, M.H. Chen, R.P. Hebbel, R.T. Tranquillo (2014) Blood outgrowth endothelial cells alter remodeling of completely biological engineered grafts implanted into the sheep femoral artery. J Cardiovasc Transl Res 7: 242-249.
Montoya, C.V., P.S. McFetridge (2009) Preparation of ex vivo-based biomaterials using convective flow decellularization. Tissue Eng Part C Methods 15: 191-200.

Olausson, M., P.B. Patil, V.K. Kuna, P. Chougule, N. Hernandez, K. Methe, C. Kullberg-Lindh, H. Borg, H. Ejnell, S. Sumitran-Holgersson (2012) Transplantation of an allogeneic vein bioengineered with autologous stem cells: a proof-of-concept study. Lancet 380: 230-237.

Orlando, G., C. Booth, Z. Wang, G. Totonelli, C.L. Ross, E. Moran, M. Salvatori, P. Maghsoudlou, M. Turmaine, G. Delario, Y. Al-Shraideh, U. Farooq, A.C. Farney, J. Rogers, S.S. Iskandar, A. Burns, F.C. Marini, P. De Coppi, R.J. Stratta, S. Soker (2013) Discarded human kidneys as a source of ECM scaffold for kidney regeneration technologies. Biomaterials 34: 5915-5925.

Pellegata, A.F., M.A. Asnaghi, I. Stefani, A. Maestroni, S. Maestroni, T. Dominioni, S. Zonta, G. Zerbini, S. Mantero (2013) Detergent-enzymatic decellularization of swine blood vessels: insight on mechanical properties for vascular tissue engineering. BioMed Res Int 2013: 918753.

Pellegata, A.F., M.A. Asnaghi, S. Zonta, G. Zerbini, S. Mantero (2012) A novel device for the automatic decellularization of biological tissues. Int J Artif Organs 35: 191-198.

Piola, M., M. Soncini, F. Prandi, G. Polvani, G. Beniamino Fiore, M. Pesce (2012) Tools and procedures for ex vivo vein arterialization, preconditioning and tissue engineering: a step forward to translation to combat the consequences of vascular graft remodeling. Recent Pat Cardiovasc Drug Discov 7: 186-195.

Price, A.P., L.M. Godin, A. Domek, T. Cotter, J. D’Cunha, D.A. Taylor, A. Panoskaltsis-Mortari (2015) Automated decellularization of intact, human-sized lungs for tissue engineering. Tissue Eng Part C Methods 21: 94-103.

Quint, C., M. Arief, A. Muto, A. Dardik, L.E. Niklason (2012) Allogeneic human tissue-engineered blood vessels. J Vasc Surg 55: 790-798.

Quint, C., Y. Kondo, R.J. Manson, J.H. Lawson, A. Dardik, L.E. Niklason (2011) Decellularized tissue-engineered blood vessel as an arterial conduit. Proc Natl Acad Sci USA 108: 92149219.

Roh, J.D., R. Sawh-Martinez, M.P. Brennan, S.M. Jay, L. Devine, D.A. Rao, T. Yi, T.L. Mirensky, A. Nalbandian, B. Udelsman, N. Hibino, T. Shinoka, W.M. Saltzman, E. Snyder, T.R. Kyriakides, J.S. Pober, C.K. Breuer (2010) Tissue-engineered vascular grafts transform into mature blood vessels via an inflammationmediated process of vascular remodeling. Proc Natl Acad Sci USA 107: 4669-4674.

-Seifu, D.G., A. Purnama, K. Mequanint, D. Mantovani (2013) Small-diameter vascular tissue engineering. Nat Rev Cardiol 10: 410-421.

Struecker, B., A. Butter, K. Hillebrandt, D. Polenz, A. Reutzel-Selke, P. Tang, S. Lippert, A. Leder, S. Rohn, D. Geisel, T. Denecke, K. Aliyev, K. Johrens, N. Raschzok, P. Neuhaus, J. Pratschke, I.M. Sauer (2014) Improved rat liver decellularization by arterial perfusion under oscillating pressure conditions. J Tissue Eng Regen Med, Epub ahead of print.

Struecker, B., K.H. Hillebrandt, R. Voitl, A. Butter, R.B. Schmuck, A. Reutzel-Selke, D. Geisel, K. Joehrens, P.A. Pickerodt, N. Raschzok, G. Puhl, P. Neuhaus, J. Pratschke, I.M. Sauer (2015) Porcine liver decellularization under oscillating pressure conditions - a technical refinement to improve the homogeneity of the decellularization process. Tissue Eng Part C Methods 21: 303-313.

Sullivan, D.C., S.H. Mirmalek-Sani, D.B. Deegan, P.M. Baptista, T. Aboushwareb, A. Atala, J.J. Yoo (2012) Decellularization methods of porcine kidneys for whole organ engineering using a high-throughput system. Biomaterials 33: 7756-7764.

Syedain, Z.H., L.A. Meier, M.T. Lahti, S.L. Johnson, R.T. Tranquillo (2014) Implantation of completely biological engineered grafts following decellularization into the sheep femoral artery. Tissue Eng Part A 20: 1726-1734.

Tara, S., H. Kurobe, M.W. Maxfield, K.A. Rocco, T. Yi, Y. Naito, C.K. Breuer, T. Shinoka (2015) Evaluation of remodeling process in smalldiameter cell-free tissue-engineered arterial graft. J Vasc Surg 62: 734-743.

Tresoldi, C., A.F. Pellegata, S. Mantero (2015) Cells and stimuli in small-caliber blood vessel tissue engineering. Regen Med 10: 505-527.

Weber, B., P.E. Dijkman, J. Scherman, B. Sanders, M.Y. Emmert, J. Grunenfelder, R. Verbeek, M. Bracher, M. Black, T. Franz, J. Kortsmit, P. Modregger, S. Peter, M. Stampanoni, J. Robert, D. Kehl, M. van Doeselaar, M. Schweiger, C.E. Brokopp, T. Walchli, V. Falk, P. Zilla, A. Driessen-Mol, F.P. Baaijens, S.P. Hoerstrup (2013) Off-the-shelf human decellularized tissue-engineered heart valves in a non-human primate model. Biomaterials 34: 7269-7280.

-Wolf, M.T., K.A. Daly, J.E. Reing, S.F. Badylak (2012) Biologic scaffold composed of skeletal muscle extracellular matrix. Biomaterials 33: 2916-2925. 